\title{
Rhinosporidiosis: Various Presentations and Different Sites
}

\author{
Saumendra Nath Bandyopadhyay, ${ }^{1}$ Utpal Jana, ${ }^{2}$ Gautam Bandyopadhyay, ${ }^{3}$ Tapas Kumar Majhi, ${ }^{4}$ Shubhrakanti Sen, ${ }^{5}$ \\ Soumyarup Das, ${ }^{6}$ Ipsita Mandal $^{7}$
}

\begin{abstract}
$\underline{\text { Introduction }}$
Rhinosporidiosis commonly affects nasal mucosa but may have varied presentations depending upon the site of involvement. Materials and Methods

119 patients of rhinosporidiosis, attending the two medical colleges of West Bengal over a period of two years were reviewed. $\underline{\text { Results }}$

Granulomatous lesion in the nose and nasopharynx was the commonest presentation. The most common symptoms were nasal obstruction, bleeding from the nose and nasal discharge. The less commonly involved sites were the eye, penis, skin, subcutaneous tissue, muscle and bone.

Discussion

Most of the extranasal rhinosporisdiosis were secondary to nasal disease. Extranasal lesions do not have the typical granular polypoid appearance of the nasal and nasopharyngeal rhinosporidiosis. Extranasal rhinosporidiosis could be excised with minimal operative bleeding.

Conclusion

Rhinosporidiosis has nasal and extranasal presentations. This chronic disease may also present acutely with respiratory distress or haemorrhage. A high degree of suspicion helps the diagnosis of extranasal rhinosporidiosis. FNAC helps in the diagnosis. Histopathology is confirmatory.

$\underline{\text { Keywords }}$

Rhinosporidiosis; Rhinosporidium; Diagnosis, Differential; Nose; Conjunctiva; Urethra; Skin; Subcutaneous Tissue; Bone, Polyps
\end{abstract}

ABSTRACT

$\mathrm{R}$ hinosporidiosis is endemic in India and Sri Lanka. This chronic granulomatous disease usually affects the nasal mucosa but atypical presentations due to involvement of other sites are not uncommon and may cause a diagnostic dilemma. This study was undertaken to document different presentations

1 - Department of ENT, Medical College, Kolkata

2 - Departtment of ENT, Sagar Dutta Medical College,

Kamarhati

3- Department of Pathology, R G Kar Medical College,

Kolkata

4- Department of Urology, NRS Medical College, Kolkata

5- Department of ENT, NRS Medical College, Kolkata

6- Dum Dum Specialized Municipality Hospital

7- Kanchrapara ESI Clinic

Corresponding author:

Dr Saumendra Nath Bandyopadhyay

email: sban_kolkata@rediffmail.com of rhinosporidiosis and their management with review of the relevant literature.

\section{Materials and Methods}

A total of 119 patients of rhinosporidiosis attending the Departments of ENT of the participating medical colleges during a period of two years (December, 2011 to November, 2013) were included for this study. Nasal Endoscopy was done in all cases of nasal presentations. FNAC was done in all suspected extranasal lesions. In a few selected cases CT Scan is advised to note the extent of lesion. All excised specimen were routinely sent for histopathological confirmation.

\section{Results}

The most common presenting symptoms of rhinosporidiosis were nasal obstruction $(87 \%)$ followed 
by bleeding from the nose $(78 \%)$ while $30 \%$ of patients complained of sticky white nasal discharge (Table I). The extranasal lesions had either a coexisting nasal rhinosporidiosis (Fig.1). Out of the 114 nasal and nasopharyngeal lesions, $67(60 \%)$ had pedunculated lesion and the rest $47(40 \%)$ had sessile or mixed

Table I: Symptoms of the Patients Suffering from Rhinosporidiosis $(n=119)$

\begin{tabular}{|c|c|}
\hline SYMPTOMS & 104 \\
\hline Nasal obstruction & 93 \\
\hline Nose bleed & 36 \\
\hline Nasal discharge & 03 \\
\hline Mass on the eyelid & 03 \\
\hline Fleshy mass on the penis & 02 \\
\hline Nodules/ warts on the skin & 01 \\
\hline Fungating mass with ulceration on the skin & 01 \\
\hline Subcutaneous swelling & 03 \\
\hline Difficulty in breathing & 03 \\
\hline Muffled speech & 02 \\
\hline
\end{tabular}

mass or history of excision of nasal rhinosporidiosis in the past, except in three cases. One of the three primary extranasal rhinosporidiosis presented with a mass coming out through the urethral opening and the other two were conjunctival rhinosporidiosis. Three patients with pedunculated mass hanging into the oropharynx had muffled speech. The lone patient of laryngeal rhinosporidiosis presented with sensation of lump in the throat. The single patient of disseminated rhinosporidiosis, in addition to the nasal mass, had subcutaneous swellings, cutaneous nodules and ulcerated growths that bled on touch, has been reported. ${ }^{1}$ The different anatomical sites involved by rhinosporidiosis in this series have been noted in Table II.

Eighty three patients were male and thirty six female; the male to female ratio being $2.3: 1$. The peak incidence was between $11-20$ years of age (17\%), followed by the 21-30 years age group (13\%). The youngest patient was a 9 year old boy while the oldest was a 78 year old man.

Most of the nasal lesions had pink fleshy mass with white dots on the surface, which is typical of crops of some sessile and some pedunculated lesions (Fig.2). Larger masses hanging into the oropharynx, on the contrary, had the look of fleshy polyps with smooth lobulated surfaces without the history of recurrent episodes of bleeding (Fig.1). 83 of the nasal/ nasopharyngeal lesions (73\%) were single and multiple fleshy masses were seen in the rest $31(27 \%)$. Floor of the nasal cavity and the nasal septum were found to be the sites most commonly involved.

The conjunctival lesions were pink globular mass with finely irregular surface, attached to the lower palpebral conjunctiva with a short narrow stalk. There was white discharge from the affected eye (Fig.3).

The cutaneous lesions were either small, non-tender nodules or warty dark coloured excrescences with small pedicles (Fig.4). Ulcerated skin lesions had fungating irregular surface, which bled on touch. The growths were firm, mildly tender and had everted margins (Fig.5). These lesions were difficult to distinguish from soft tissue malignancy.

The subcutaneous and intramuscular lesions were 
Table II: Anatomical Sites Involved by Rhinosporidiosis ( $=119)$

\begin{tabular}{|c|c|}
\hline INVOLVED SITES * & 114 \\
\hline Nose and nasopharynx & 2 \\
\hline Conjunctiva & 1 \\
\hline Lacrimal sac & 3 \\
\hline Urethra/ Glans penis & 1 \\
\hline Larynx & 2 \\
\hline Skin & 1 \\
\hline Bone & 1 \\
\hline Subcutaneous tissue & 1 \\
\hline Muscle & \\
\hline
\end{tabular}

of two types, firm and cystic. The firm lesions were lobulated, pale yellow with homogenous cut surface, which looked similar to brain tissue (Fig.6). The single cystic ovoid intramuscular lesion had smooth surface with a thick fibrous capsule (Fig.7) and contained about $20 \mathrm{ml}$ of thick creamy fluid, which, on microscopy, showed presence of numerous sporangia of different stages of maturation (Fig.8). The fibrous capsule, on the other hand, did not show any presence of granulation or spore. ${ }^{1}$

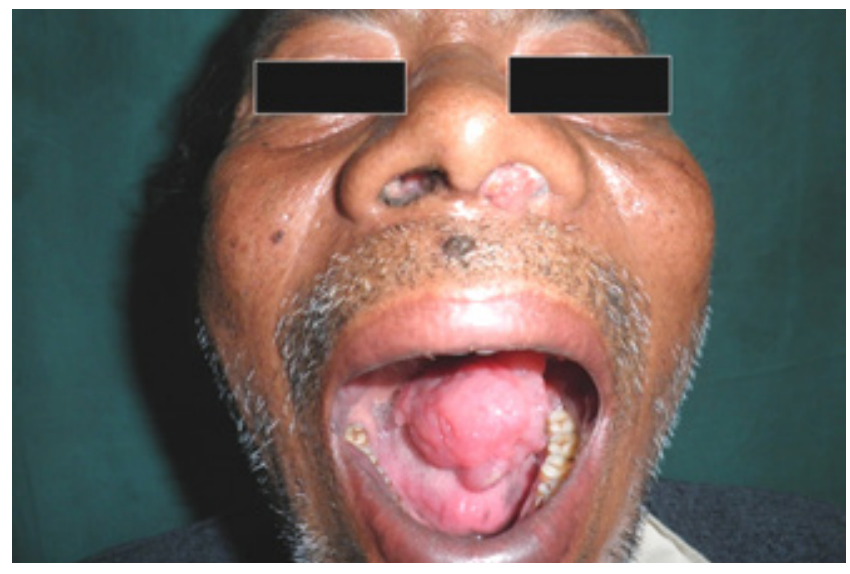

Fig. 1 Rhinosporidial mass filling up the left nasal cavity and the polypoid oropharyngeal extension brought into the mouth of the patient
The involvement of penis presented either as discrete pink polypoidal mass with granular surface, protruding from the urethral meatus or as a smooth globular sessile mass arising from the glans penis (Fig.9).

Involvement of the bone in rhinosporidiosis presented as almost painless swelling over the wrist joint with restriction of movement. ${ }^{1}$ The skin covering the swelling got ulcerated and grew to form a huge fungating ulceroproliferative mass (Fig.10).

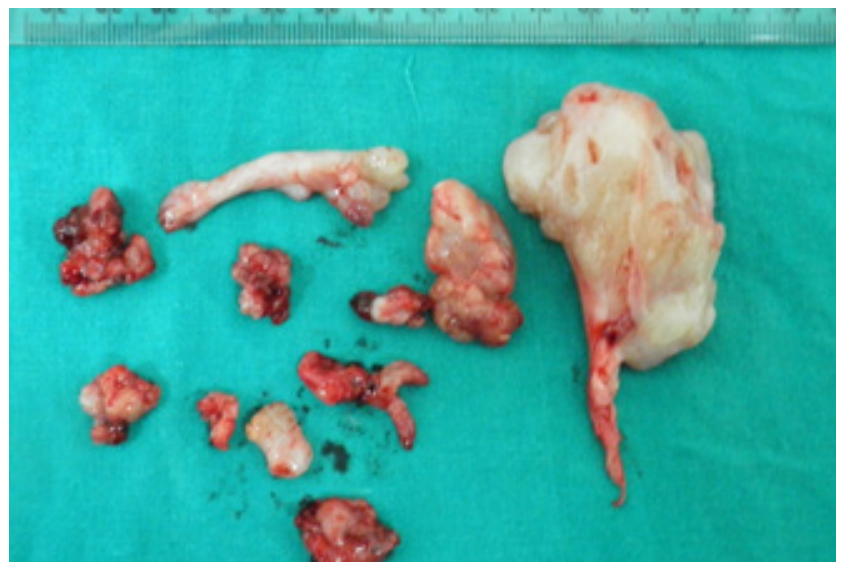

Fig. 2 Multiple polypoidal and sessile rhinosporidial mass removed from a single patient 


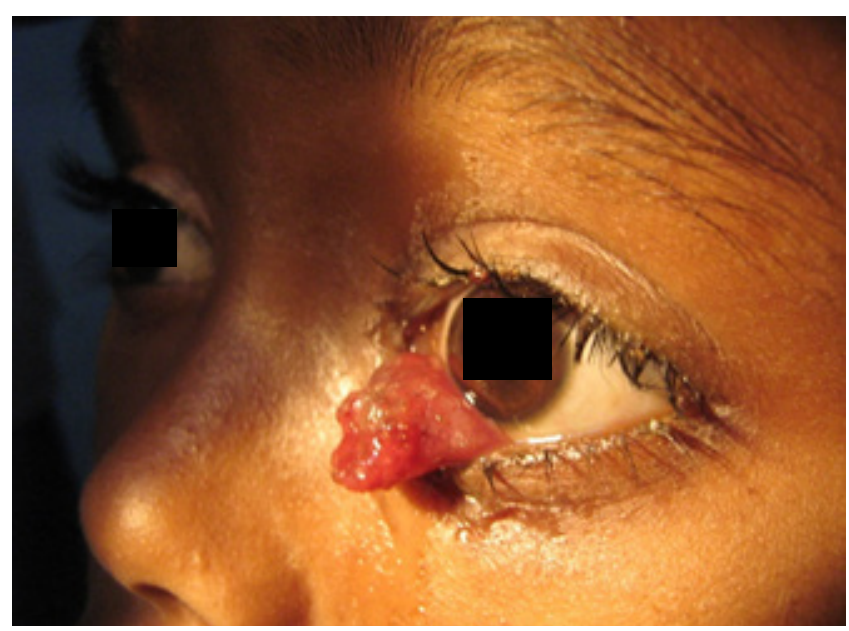

Fig. 3 Conjunctival rhinosporidiosis
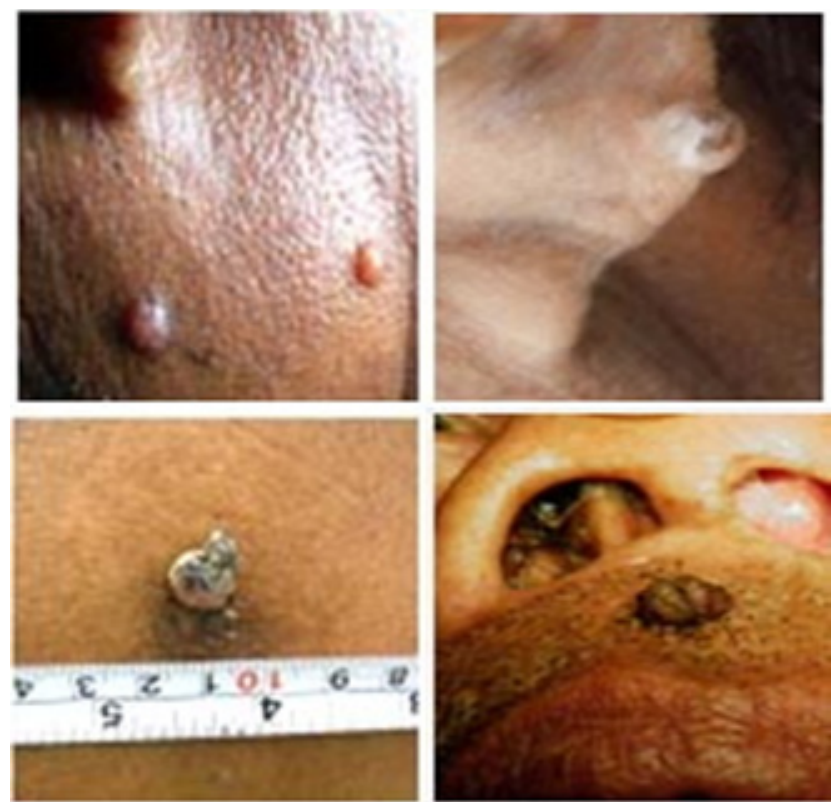

Fig. 4 Cutaneous lesions of rhinosporidiosis: (clockwise from top left) nodules on cheek, nodule on the pinna, warty lesion on the upper lip and warty lesion with a small pedicle on the back

The laryngeal lesion was a solitary pink polyp attached to the left aryepiglottic fold with a short stalk.

None of the cases in this series showed any enlargement of the regional lymph nodes.

Radiologically rhinosporidiosis appeared as an inhomogeneously enhancing or hypodense SOL in CT scan, usually occupying the nose and nasopharynx.
Bone erosion was not usually seen in rhinosporidiosis. Erosion of bony nasal septum was seen in one case. Turbinates were often seen to be incorporated in the nasal mass. Paranasal sinuses were never found to get involved. The single case of involvement of the distal end of the radius showed multiple osteolytic lesions (Fig.11).

FNAC from the subcutaneous swellings showed presence of sporangia in different stages of maturation and a few foreign body type giant cells (Fig.12). Histopathological diagnosis has been considered to be confirmatory in this series. All the excised masses were subjected to histopathological examination. Staining with $H \& E$ revealed multiple mature and immature sporangia of sizes $50-100 \mu \mathrm{m}$ diameter packed with

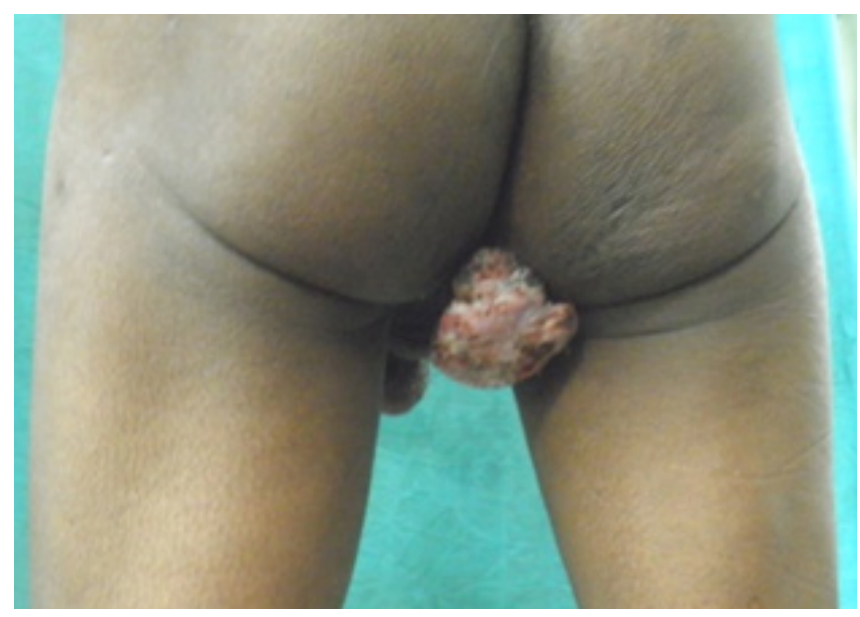

Fig. 5 Fungating mass arising from the perianal skin

spores. A few plasma cells and lymphocytes were seen in the stroma (Fig.13).

Treatment of rhinosporidiosis was tailored according to the stage and mode of presentation. When patients presented with bleeding or an infected mass, pressure bandaging (for lesions on the surface) or nasal packing was applied where possible, to minimize bleeding. Whole blood transfusion was given in some cases. Antibiotics were needed to control infection. Preliminary tracheostomy was done in three cases who presented with respiratory distress due to huge rhinosporidial mass hanging into the oropharynx, causing upper airway obstruction.

The definitive treatment, as a rule, was wide 


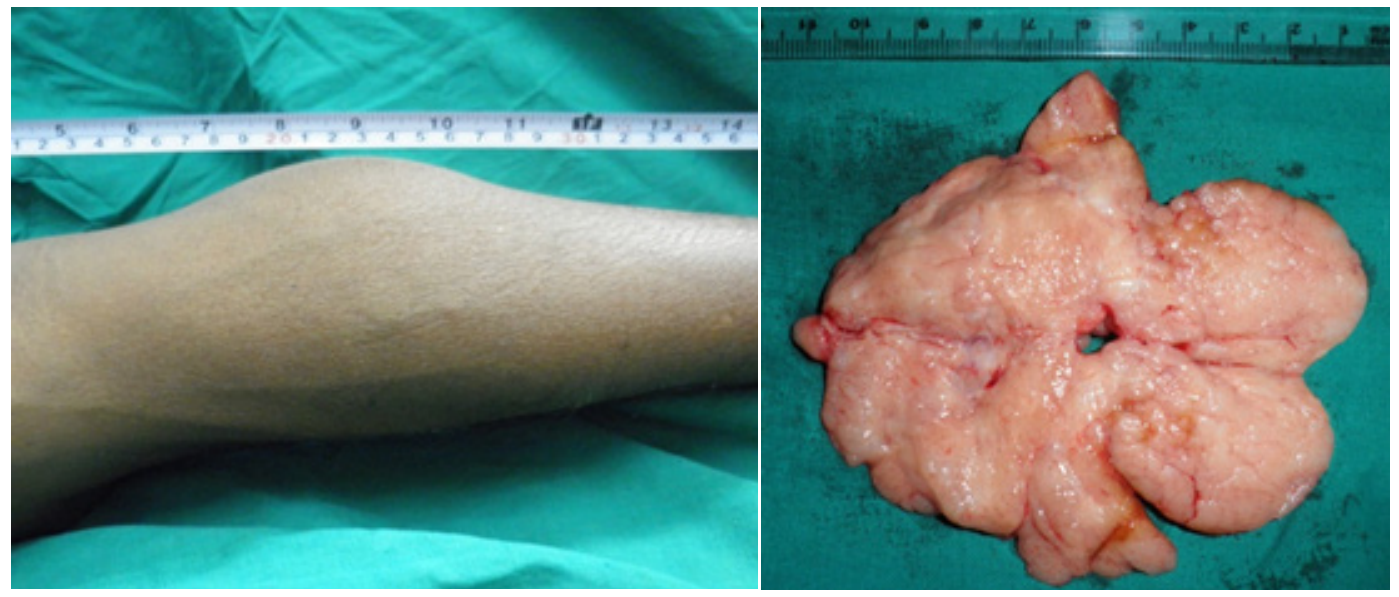

Fig. 6 (Left) Forearm with subcutaneous rhinosporidiosis. (Right) Cut surface of the excised subcutaneous rhinosporidiosis removed from the forearm

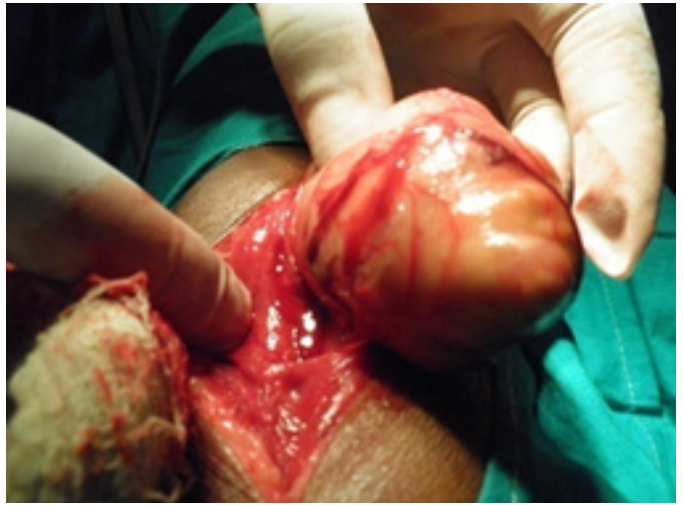

Fig. 7 Intramuscular cystic rhinosporidiosis being removed from the right calf

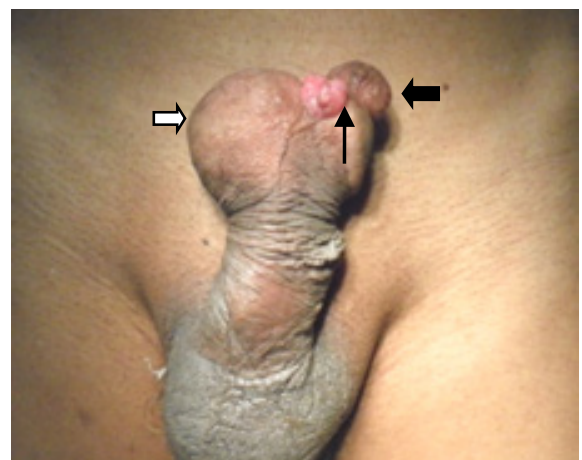

Fig. 9 The penile lesions showing the urinoma (white arrow), fleshy polypoid mass on the tip of the glans penis (bold arrow) and its under-surface (thin arrow)

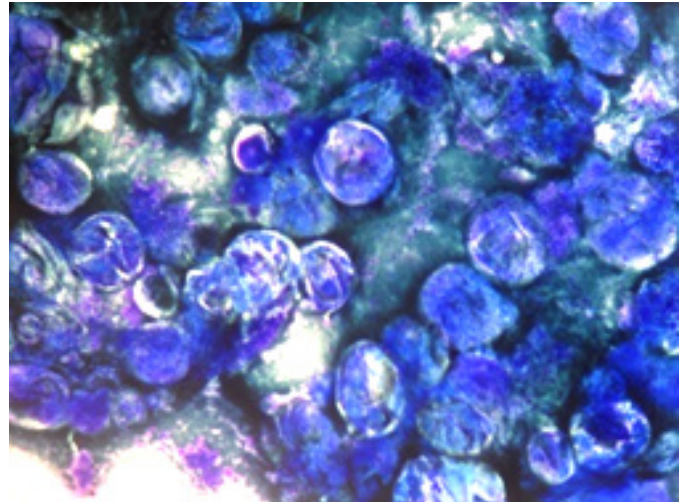

Fig. 8 Centrifuged deposit from cyst fluid showing numerous sporangia MGG $\times 40$

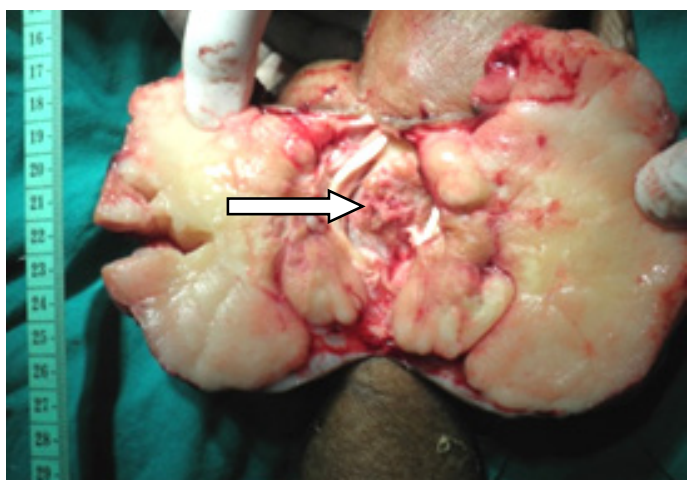

Fig. 10 The amputation specimen showing the cut surfaces of the growth on the wrist and involvement of the underlying bones (white arrow) 

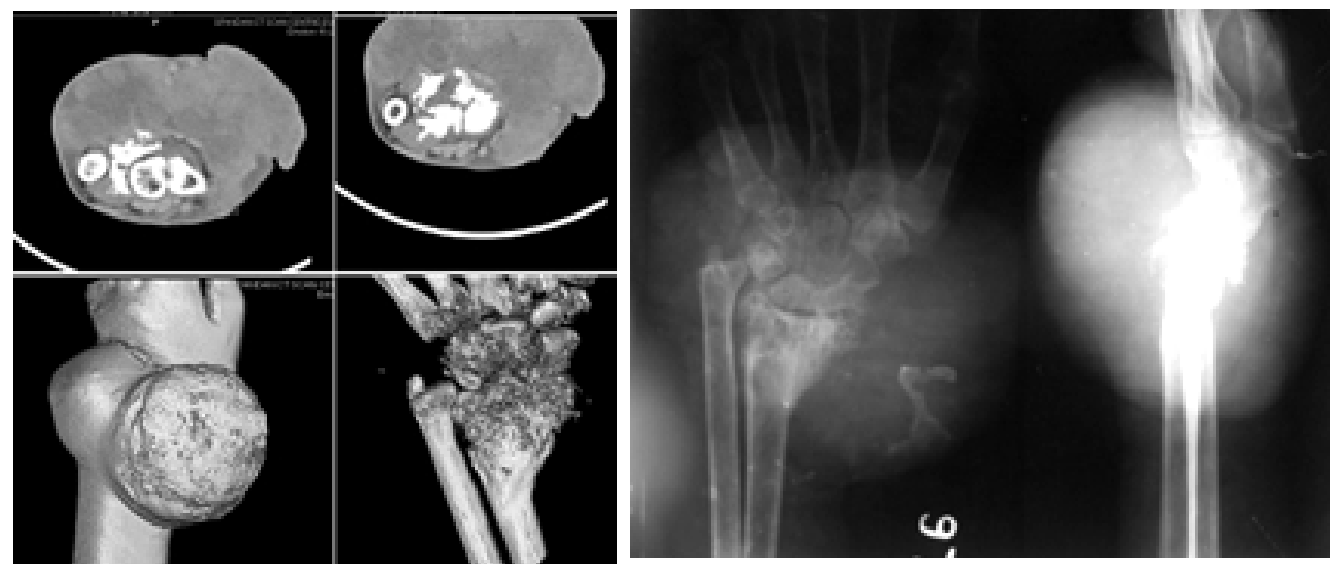

Fig. 11 X-ray of the wrist showing osteolytic lesions affecting the lower ends of the radius and ulna. CT scan windows showing the extent of the soft tissue and bone involvement

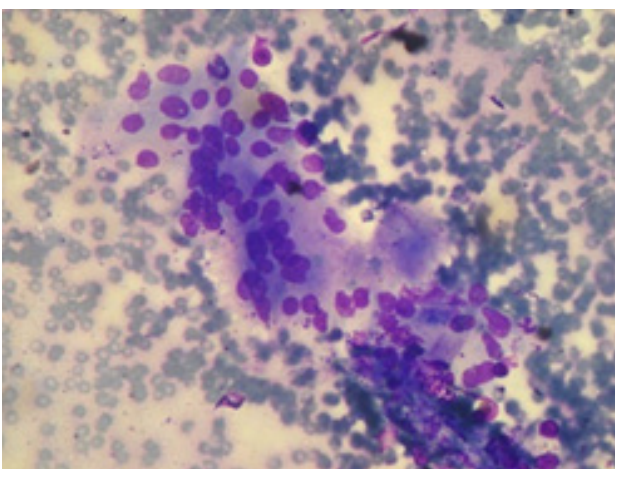

Fig. 12 Giant cell reaction with a ruptured sporangium having spores within, FNAC, MGGx100

excision with diathermy coagulation of the base. Nasal endoscopy helped in dealing with the difficult-to-access lesions arising from the posterior part of the nasal cavity and nasopharynx. Lesions with multiple attachments or excessive operative bleeding were subjected to repeat endoscopy three weeks later to cauterize the granular areas on the mucosa. The subcutaneous lesions were enucleated from the subfascial or intramuscular locations with primary closure of the skin incision. These lesions had smooth glistening surfaces and could be easily enucleated with very little bleeding. Involvement of bone or joint necessitated partial amputation with wide margin as the marrow spaces were invaded by rhinosporidiosis. Genitourinary rhinosporidiosis involving the distal part of the penile urethra and the navicular fossa could be approached by ventral meatotomy and excision of the mass with diathermy coagulation of the base to prevent

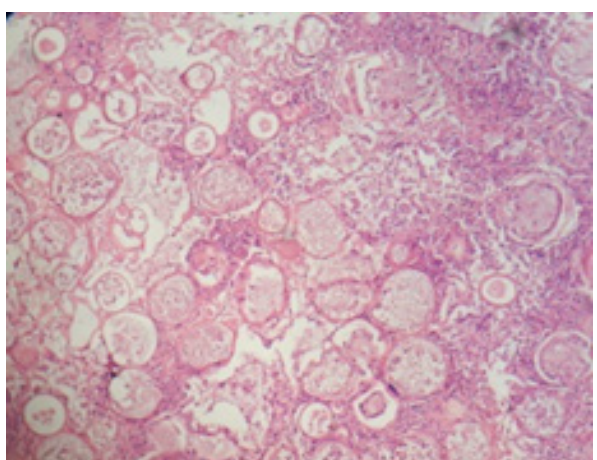

Fig. 13 Histopathological picture of rhinosporidiosis showing cysts of variable sizes, containing numerous spores in different stages of development, H\&E X40

recurrence. Involvement of the glans penis required partial amputation of penis.

\section{Discussion}

Rhinosporidium seeberi, the causative agent of rhinosporidiosis, is a eukaryote pathogen, a protoctistan parasite of the class Mesomycetozoea., ${ }^{2,3}$ The infective stage of the organism is the functional spore, which is approximately $10-12 \mu$ in diameter. ${ }^{4}$ Definite mode of transmission, host and natural reservoirs are largely unknown but the disease is presumed to be transmitted by direct contact with spores through dust, infected clothing or fingers and bathing in stagnant water. ${ }^{5,6}$ This explains the predilection of infection for mucosal sites, where the organism gains access through traumatized epithelium. ${ }^{5}$ Over $70 \%$ of the reported cases involved the 
nasal mucosa and about $15 \%$ were in the eye. ${ }^{6} 95 \%$ of patients in this series presented with nasal involvement. Ocular and genitourinary involvement came next with involvement in $2.5 \%$ each.

Ocular rhinosporidiosis is usually airborne., ${ }^{4,7}$ Ascending infection from the nose is implicated for involvement of the lacrimal sac. Occasional involvement of lips, palate, epiglottis, pharynx, larynx, trachea, bronchus, ears, conjunctiva, lacrimal sac, skin, vulva, vagina, penis, scalp and bone has also been reported. ${ }^{8,9}$ Rhinosporidiosis is endemic in south Asia and approximately $90 \%$ of all reported cases were from India and Sri Lanka., ${ }^{9}$

The most common presenting symptom of rhinosporidiosis are nasal mass, nasal obstruction, epistaxis and nasal discharge. ${ }^{4,11}$ Other symptoms may vary depending upon the site of infection. Oral mass, ocular mass, haemoptysis, swelling over bone or joint, nodules or warts on the skin or genitalia, ulcerative lesion or ulceroproliferative growth may be some of the other presentations. ${ }^{1,4,6,9,12,13,14,15}$

The typical look of rhinosporidiosis is usually sufficient to arrive at a diagnosis. But delayed presentation, extranasal presentation or involvement of multiple anatomically unrelated sites make the diagnosis difficult. Acute presentation of this chronic disease has also been noted. Three of the patients had to undergo preliminary tracheostomy to relieve respiratory obstruction caused by huge oropharyngeal mass hanging down from the nasopharynx.

The oropharyngeal masses lacked the typical look of rhinosporidiosis. The fleshy pink or pale lobulated oropharyngeal masses may look like antrochoanal polyp or inverted papilloma. ${ }^{4}$ The possibility of angiofibroma should also be considered for a nasopharyngeal mass with history of epistaxis. Involvement of other parts of the respiratory tract is usually secondsary to nasal rhinosporidiosis. The single case of laryngeal involvement in this series presented with sensation of lump in the throat. There was history of bleeding par mouth. The patient had history of surgeries for nasal rhinosporidiosis twice in the last three years. It presented with a small fleshy mass on the arytenoid with granular surface.
The cutaneous lesions, found in patients with previous surgery for rhinosporidiosis, were nodular, warty or ulceroproliferative. Multiple skin coloured small nodules of less than $0.5 \mathrm{~cm}$ separated with normal intervening skin were found on the face. Slowly enlarging brownish-black warty lesions of sizes varying from less than $0.5 \mathrm{~cm}$ to $2 \mathrm{~cm}$ diameter with short pedicles were found on the skin of the upper lip and on the back. Ulceroproliferative growths were either pedunculated or sessile growth with everted margins and involvement of the underlying bone (the radius). Differential diagnoses of cutaneous rhinosporidiosis include coccidiodomycosis, ${ }^{9}$ adiaspiromycosis, ${ }^{9}$ wart, haemangioendothelioma, ${ }^{15}$ sarcoma or other soft tissue tumours.

Subcutaneous firm swellings of rhinosporidiosis were excised from subcutaneous, subfascial or intramuscular locations. ${ }^{1}$ These lesions were not vascular and could be excised with minimal bleeding. Subcutaneous rhinosporidiosis masses have cerebriform, cream coloured appearance, with homogeneous pale yellow cut surfaces and the consistency of the brain tissue. ${ }^{12}$ In one of the cases in this series, the surface of a mass removed from the subfascial plane of the anterior chest wall was finely granular and surprisingly enough, one intramuscular lesion was cystic. ${ }^{1}$ The cyst contained about $20 \mathrm{ml}$ of thick cream coloured fluid. The wall of the cyst was fibrous and thick, with no evidence of granuloma or sporangia in it. The fluid was teeming with sporangia (Fig. 8).

Osteolytic lesions of rhinosporidiosis involving tibia,${ }^{6,8}$ fibula, ${ }^{6}$ femur, ${ }^{16}$ scapula, ${ }^{17}$ hand and foot, ${ }^{8,18}$ talus ${ }^{6}$ and calcaneum, ${ }^{6,15}$ have been reported.

Excision $^{17,19}$ or partial amputation, ${ }^{6,8}$ are the suggested treatment modalities. Extensive soft tissue involvement, infiltration of the marrow space and involvement of the wrist joint prompted us to opt for a below-elbow amputation to ensure adequate disease-free margin.

Diagnosis of rhinosporidiosis is possible with FNAC and staining the aspirate with MGG, PAP or PAS. The biological agent has a mature stage that consists of large thick walled spherical structures called sporangia and smaller daughter cells called endospores. FNAC from the subcutaneous swellings in our patient showed 
abundant intact sporangia filled with endospores (Fig.14) as well as ruptured sporangia with dispersed spores and few foreign body type giant cells (Fig.15). However a definitive diagnosis of rhinosporidiosis needs histopathological identification of the pathogen on the biopsied specimen in its diverse stages of maturation, ${ }^{9}$ which is possible with standard H\&E staining.

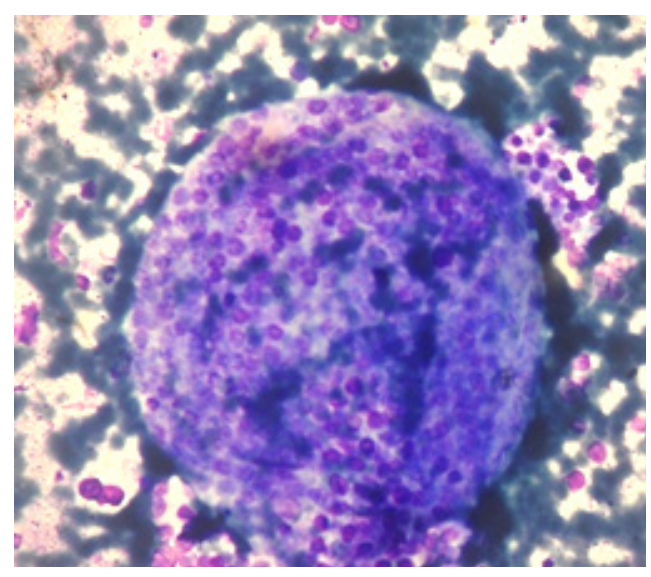

Fig. 14 Intact sporangium with numerous spores, FNAC, MGG $x 400$

Both recurrence and residual diseases are common in rhinosporidiosis. Wide excision of the lesion with diathermy coagulation of the base to prevent spread through the submucosal lymphatics is the definitive treatment of rhinosporidiosis. Complete excision sometimes becomes difficult in sessile lesions or when the lesions are located in the posterior part of the nasal cavity and nasopharynx. Brisk bleeding associated with

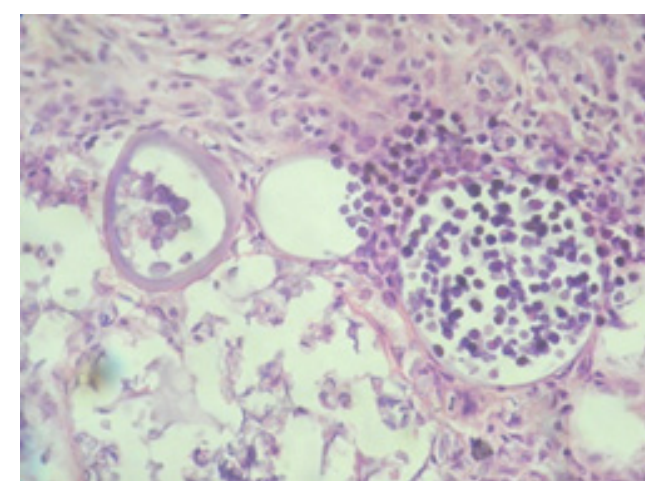

Fig. 15 High power view of an intact and ruptured sporangium containing numerous spores $H \& E$ x 100 surgeries may also be a cause of incomplete removal. Seeding of the operative sites with the spores present on the surface of the granuloma may also contribute to the recurrence of the lesion. The lifestyle and personal habit of the patient are also contributory factors. Haematogenous spread may be responsible for dissemination of the disease. ${ }^{1,6,18}$ Eleven cases $(9 \%)$ in this series were operated for recurrent disease and five $(4 \%)$ of them had multiple recurrences. We tried to localize residual disease by performing nasal endoscopy three weeks post-op, when there was excessive operative bleeding or multiple attachments detected during the primary operation. All the patients were advised review every two months. Six of the patients, who were operated during the study period, had recurrence of nasal lesions within one year after operation. The actual recurrence rate may be higher because as many as thirty six patients $(30 \%)$ were lost in the follow up. Patients with thick nasal discharge, history of previous operation, excessive operative bleeding and attachments in the difficult to access areas were the main reasons for recurrences. Use of KTP Laser, ${ }^{20}$ Diode Laser, ${ }^{21}$ Harmonic scalpel, ${ }^{22}$ and coblation $^{23}$ has been mentioned in the literature to help minimize bleeding and recurrences.

Though recurrence of rhinosporidiosis is very common, dissemination of the disease is very rare. Only a few cases of disseminated rhinosporidiosis with involvement of anatomically unrelated distant sites like subcutaneous tissue, bone, urethra and widespread lesion on the skin have been reported. ${ }^{6,8,9,12,15,17,18,24}$ One of the patients reviewed in this series had disseminated rhinosporidiosis with involvement of nasal and extranasal sites. ${ }^{1}$

Dapsone $(100 \mathrm{mg} /$ day) has traditionally been used in the medical management of rhinosporidiosis, especially in recurrent and unresectable cases but the results so far has not been good. Orally administered drugs can take longer to reach the affected areas in the presence of haemorrhage, oedema and inflammation. Wherever possible, surgical excision followed by drug therapy would be more effective. ${ }^{25,26}$ Multi-drug therapy using cycloserine, ketoconazole and dapsone, has recently been recommended for treating disseminated rhinosporidiosis, not responding to dapsone monotherapy. ${ }^{27}$ We have used dapsone for the patient 
of disseminated rhinosporidiosis in the postoperative period and the patient did not have any recurrence in the one year follow up period.

\section{Conclusion}

Nasal and nasopharyngeal mucosa are most commonly involved in rhinosporidiosis. The typical red, granular friable polypoid appearance may not be evident in extranasal rhinosporidiosis. Involvement of extranasal sites can make the diagnosis of rhinosporidiosis difficult. A high degree of suspicision may help in the diagnosis of such presentations. This chronic disease may also present with acute presentation like respiratory obstruction and haemorrhage. FNAC may help the diagnosis. Histopathology is confirmatory.

\section{References}

1. Bandyopadhyay SN, Das S, Majhi TK, Bandyopadhyay G, Roy D. Disseminated rhinosporidiosis. J laryngol Otol 2013; 127: 1020-4. doi:10.1017/S0022215113002193

2. Herr RA, Ajello L, Lepp PW et al. Phylogenetic analysis of Rhinosporidium seeberi's 18S small-subunit ribosomal DNA groups this pathogen among members of the protoctistan mesomycetozoea class. J Clin Microbiol 1999;37:2750-4

3. Vilela R, Mendoza L.The taxonomy and phylogenetics of the human and animal pathogen Rhinosporidium seeberi: a critical review.Rev Iberoam Micol. 2012; 29:185-99. doi: 10.1016/j. riam.2012.03.012

4. Venkatachalam VP, Anand N, Bhooshan O. Rhinosporidiosis: Its varied presentations. Indian J Otolaryngol Head Neck Surg 2007; 59: 142-4

5. Azad NS, Ahmad Z, Kayani N. Rhinosporidiosis presenting as an urethral polyp. J Coll Physicians Surg Pakistan 2008; 18: 314-5

6. Amritanand R, Nithyananth M, Cherian VM, Venkatesh K. Disseminated rhinosporidiosis destroying the talus: a case report. J Ortho Surg 2008;16:99-101

7. Ratnakar C, Madhavan M, Sankaran V, Veliath AJ, Majumdar NK, Rao VA. Rhinosporidiosis in Pondicherry. J Trop Med Hyg 1992; 95: 71-2

8. Gokhale S, Ohri VC, Subramanya H, Reddy PS, Sharma SC. Subcutaneous and osteolytic rhinosporidiosis. Indian J Pathol Microbiol 1997;40:95-8

9. Anoop TM, Rajany A, Deepa PS, Sangamithra P, Jayaprakash R. Disseminated cutaneous rhinosporidiosis. J R Coll Physicians Edinb 2008;38:123-5

10. Moses JD, Shanmugham A. Epidemiological survey of rhinosporidiosis in man - A sample survey in a high school located in a hyperendemic area. Indian Veterinary Journal 1987; 64: 34-8

11. Das BC. Rhinosporidiosis. Indian J Otol 1974; 26: 79-84

12. Angunawela P, De Tissera A, Dissanaike AS. Rhinosporidiosis presenting with two soft tissue tumors followed by dissemination. Pathology 1999;31:57-8

13. Sarker Md Moniruzzaman, Kibria AKM Golam, Haque Md Manzurul. Disseminated subcutaneous rhinosporidiosis: A case report. The Journal of Teachers association RMC, Rajshahi 2006;19:31-3

14. Pal DK, Moulik D, Chowdhury MK. Genitourinary rhinosporidiosis. Indian J Urol 2008;24:419-21

15. Mitra K, Maity PK. Cutaneous rhinosporidiosis. J Indian Med Assoc 1996;94:84

16. Dash A, Satpathy S, Devi K, Das BP, Dash K. Cytological diagnosis of rhinosporidiosis with skeletal involvement: a case report. Indian J Pathol microbiol 2005;48:215-7

17. Aravindan KP, Viswanathan MK, Jose L. Rhonosporidioma of bone - a case report. Indian J Pathol Microbiol 1989;32:312-3

18. Chatterjee PK, Khatua CR, Chatterjee SN, Dastidar N Recurrent multiple rhinosporidiosis with osteolytic lesions in hand and foot. A case report. J Laryngol Otol 1977;91:729-34

19. Adiga BK, Singh N, Arora VK, Bhatia A, Jain AK. Rhinosporidiosis. Report of a case with an unusual presentation with bony involvement. Acta Cytol 1997;41:889-91

20. Kameswaran M, Anand Kumar RS, Murali S, Raghunandhan S, Jacob J. KTP-532 laser in the management of rhinosporidiosis. Indian J Otolaryngol Head Neck Surg 2005; 57: 298-300.doi: 10.1007/BF02907692.

21. Nichlani S., Jagade M. V., Ganeshan A. P., Sayeed S., Borade A. Endoscopic resection of a nasal rhinosporidiosis with diode Laser. Bombay Hospital Journal.2011;53(1):96-8

22. Chery J, Bacskai C, Mendoza E. Recurrent rhinosporidiosis. Journal of Medical Cases. 2014;5:58-61.

23. Khan I, Gogia S, Agarwal A, Swaroop A. Recurrent Rhinosporidiosis: Coblation Assisted Surgical Resection-A Novel Approach in Management. Case Rep Otolaryngol. 2014; 2014: 609784.Published online 2014 Dec 10. doi: $10.1155 / 2014 / 609784$

24. Kumari R, Laxmisha C, Thappa DM. Disseminated cutaneous rhinosporidiosis. Dermatol Online J 2005;11:19

25. Arsecularatne SN. Chemotherapy of rhinosporidiosis - a review. J Infect Dis Antimicrob Agents 2009; 26: 21-7

26. Bhat V. Comments on novel multidrug therapy for disseminated rhinosporidiosis, refractory to dapsone - case report'. Trop Doct. 2014 Jan;44(1):59-60. doi: 10.1177/0049475513512635

27. George L, Dincy P, Chopra M, Agarwala M, Maheswaran S, Deodhar D, Rupali P, Thomas M, Pulimood S. Novel multidrug therapy for disseminated rhinosporidiosis, refractory to dapsone -case report . Tropical Doctor 2013; 43: 110-112. doi: $10.1177 / 0049475513493414$ 